\title{
Presentation and outcomes of necrotizing soft tissue infections
}

This article was published in the following Dove Press journal:

International Journal of General Medicine

31 July 2017

Number of times this article has been viewed

\section{Kuan-Chin Jean Chen' Michelle Klingel ${ }^{2}$ \\ Shelley McLeod ${ }^{3}$ \\ Sean Mindra ${ }^{4}$ \\ Victor $\mathrm{K} \mathrm{Ng}^{5}$}

'Department of Emergency Medicine, Faculty of Medicine, The Ottawa Hospital, University of Ottawa,

Ottawa, ${ }^{2}$ Division of Respiratory Medicine, Department of Pediatrics, Hospital for Sick Children, University of Toronto, ${ }^{3}$ Department of Family and Community Medicine, University of Toronto, Schwartz/Reisman Emergency Medicine Institute, Mount Sinai Hospital, Toronto, ${ }^{4}$ Department of Family Medicine, Faculty of Medicine, University of Ottawa, Ottawa, ${ }^{5}$ Division of Emergency Medicine, Faculty of Medicine, London Health Sciences Centre, Western University, London, ON, Canada
Correspondence: Kuan-Chin Jean Chen The Ottawa Hospital, 50I Smyth Road, Ottawa, ON KIH 8L6, Canada Tel +l 6I37985555

Email jeanchen789@gmail.com
Background: Necrotizing soft tissue infections (NSTIs) are aggressive infections associated with significant morbidity, including amputation and organ failure, and high mortality. The rapid progression and significant risk of morbidity and mortality associated with NSTIs makes quick diagnosis and treatment critical. The objective of this study was to determine the presentation of patients diagnosed with NSTIs and their in-hospital outcomes.

Methods: This was a retrospective review of adult ( $>17$ years) patients with a discharge diagnosis of necrotizing fasciitis at London Health Sciences Centre (annual census 125,000) over a 5-year period (April 2008-March 2013).

Results: Sixty patients with confirmed NSTI were included in this study. Common comorbidities at presentation included immunocompromise (58.3\%), diabetes mellitus (41.7\%), vascular disease (45.0\%), and obesity (24.6\%). Initial presentations included swelling (91.7\%), erythema (86.7\%), bullae $(28.3 \%)$, petechiae $(8.3 \%)$, and bruising $(45.0 \%)$. Fifty $(83.3 \%)$ underwent surgery, with a median (interquartile range) time from initial emergency department presentation to surgery of 15.5 hours $(7.8,74.9)$. In-hospital mortality among those who had surgical intervention was $14.0 \%$, compared to $60.0 \%$ for patients who did not have surgery $(\Delta 46.0 \%$; $95 \%$ CI: $14.8 \%$ to $70.2 \%$ ).

Conclusion: Diabetes mellitus, immune-compromise, vascular disease, and obesity are common comorbidities of NSTIs. Survival is higher among patients who receive surgical treatment. Patients presenting with this clinical picture warrant a high degree of suspicion.

Keywords: clinical evaluation and treatment, skin diseases, infectious diseases of the skin

\section{Background}

Necrotizing soft tissue infection (NSTI) is an infection of any of the layers within the soft tissue compartment with necrotizing changes. ${ }^{1}$ Left untreated, up to $100 \%$ of NSTIs can result in mortality. ${ }^{2}$ Even with aggressive treatment, including multiple surgical debridements and broad spectrum antibiotics, ${ }^{3,4}$ patients may suffer significant morbidity such as amputation and organ failure, ${ }^{5-10}$ and mortality can be as high as $29 \% .{ }^{11}$ With an estimated $90-200$ cases per year in Canada, ${ }^{12}$ NSTIs are rare. The diagnosis is challenging, as there are no known signs or symptoms of NSTIs that reliably distinguish them from non-necrotizing infections.

Previous retrospective studies have attempted to identify common presentations of NSTIs. In a small case series of 12 patients with NSTIs, Sin et al identified swelling $(92 \%)$, pain (92\%), erythema (75\%), and fever (67\%) as the most common presentations. ${ }^{13}$ Similarly, in a review of 58 cases of NSTIs, Mitchell et al reported swelling $(83 \%)$ and severe pain $(76 \%)$ as the most commonly presenting signs. ${ }^{11}$ Other clinical 
presentations include hard induration of the soft tissue, bullous lesions, skin necrosis, and crepitus. ${ }^{14-16}$ As the most common manifestations of NSTIs are relatively sensitive but are not specific, the best tool for the detection of NSTIs remains a high index of suspicion. Perhaps one of the more specific symptoms of NSTIs is pain out of proportion to the physical exam, underpinning the insidious spread of NSTIs. ${ }^{5,17}$ The gold standard for diagnosis of a NSTI, and the essential treatment, is surgery and discovery of necrotic tissue upon debridement. ${ }^{10}$ Yet, without a high index of suspicion, few clinicians will send their patients for such invasive investigation.

Recognizing NSTIs followed by quick initiation of therapy and surgical debridement are the most important factors in predicting patient outcomes. Previous studies have identified risk factors associated with NSTIs and found that patients who are immunocompromised, obese, of advanced age, or have peripheral vascular disease have a higher risk of NSTI. ${ }^{2}$ In addition, a history of trauma, surgery, or penetrating injury, however insignificant (skin abrasion, ${ }^{18}$ intramuscular injection), ${ }^{19}$ is common. ${ }^{2,10}$ Several studies have reported a high occurrence of diabetes in patients with NSTIs, comorbid in $\sim 45 \%$ of cases. ${ }^{20}$ As with the symptoms described earlier, these risk factors are nonspecific for NSTI. ${ }^{4,19}$ At least two scoring systems have been devised as tools to try and separate NSTIs from less worrisome nonNSTIs. From a population of 359 patients that included 31 with NSTIs, Wall et al developed a prognostic clinical tool to help identify patients with NSTIs. They were able to produce a model that was $90 \%$ sensitive and $76 \%$ specific for NSTIs, with a positive predictive value of $26 \%$ and a negative predictive value of $99 \%{ }^{21}$ They found that white blood cell (WBC) count $>15.4 \times 10^{9} / \mathrm{L}$ and serum sodium concentration $<135$ $\mathrm{mmol} / \mathrm{L}$ were particularly useful parameters. The Laboratory Risk Indicator for Necrotizing Fasciitis (LRINEC) scoring tool uses laboratory results for glucose, total WBC count, hemoglobin, sodium, serum creatinine, and $\mathrm{C}$-reactive protein (CRP) to predict NSTIs. A LRINEC score of $\geq 6$ has a positive predictive value of $92 \%$ and a negative predictive value of $96 \%$ for NSTIs. ${ }^{22}$ However, recent studies have questioned the validity of the LRINEC score. ${ }^{23-25}$ In a 2012 study, when applied to NSTIs affecting the neck alone, the LRINEC tool was only $56 \%$ sensitive and $60 \%$ specific. ${ }^{24}$ Wilson and Schneir report a case of confirmed NSTI with a LRINEC score of $0 .{ }^{25}$ A Taiwanese cohort study of 233 patients with NSTIs and 3155 patients with severe cellulitis found that a LRINEC score of $\geq 6$ had a positive predictive value of only $38 \%$, and a negative predictive value of $92.5 \%$. The authors concluded that this scoring system might be best for ruling out NSTIs. ${ }^{26}$

The objective of this study was to determine the presentation of patients diagnosed with NSTIs and the in-hospital patient outcomes (including antibiotic treatment, surgery, and mortality) associated with this disease.

\section{Methods}

This was a retrospective medical record review of adult (>17 years) patients with a hospital discharge diagnosis of "necrotizing fasciitis" at either of two academic tertiary care emergency departments (annual census 125,000) over the 5-year fiscal period of April 2008-March 2013. Both inpatient and emergency department visits were included. Patients transferred directly from another facility for specialty treatment were excluded. Charts were reviewed for patient demographics, comorbidities, clinical and laboratory features at initial presentation, wound and blood culture results, treatment, extent of surgical debridement, and patient outcomes (in-hospital mortality, length of stay). In-patient charts were reviewed in order to assess further characteristics or progression of NSTIs, as well as treatment and outcome data. LRINEC score and Wall criteria were assessed from laboratory results available in medical records. The LRINEC score was calculated as described previously (Table 1). ${ }^{22}$

Descriptive statistics were summarized using means with SD for normally distributed data, or medians with interquartile ranges (IQRs) for nonparametric data, and proportions for categorical data. Differences in proportions were assessed for the primary outcome of in-hospital mortality using Pearson chi-squared or Fisher's exact test. All data analyses were performed using SPSS 21.0 (IBM Corporation).

The study protocol was approved by the Health Sciences Research Ethics Board at the University of Western Ontario, London, $\mathrm{ON}$, and did not require written informed consent to be obtained from the participants, as this was a retrospective study, and all data were anonymous. Confidentiality of patient data was maintained at all times.

\section{Results}

Sixty patients with confirmed NSTI were included in the study. Mean (SD) age was 53.7 years (17.8), 60\% were male, and the median (IQR) hospital length of stay was 17.1 days $(7.8,33.5)$ (Table 2). Common comorbidities at presentation included immune compromise (58\%), diabetes mellitus (42\%), vascular disease (45\%), and obesity (25\%). Initial presentations included swelling (92\%), erythema (87\%), bullae (28\%), petechiae (8\%), and bruising (45\%). Fifty-nine (98\%) 
Table I Scoring systems assessed in this study for identifying NSTI

\begin{tabular}{|c|c|}
\hline Variable & Score \\
\hline \multicolumn{2}{|l|}{ LRINEC score } \\
\hline \multicolumn{2}{|l|}{ C-reactive protein, $\mathrm{mg} / \mathrm{L}$} \\
\hline$<150$ & 0 \\
\hline$\geq 150$ & 4 \\
\hline \multicolumn{2}{|l|}{ Total white cell count, $/ \mathrm{mm}^{3}$} \\
\hline$<15$ & 0 \\
\hline $15-25$ & 1 \\
\hline$>25$ & 2 \\
\hline \multicolumn{2}{|l|}{ Hemoglobin, g/L } \\
\hline$>135$ & 0 \\
\hline $110-135$ & 1 \\
\hline$<110$ & 2 \\
\hline \multicolumn{2}{|l|}{ Sodium, $\mathrm{mmol} / \mathrm{L}$} \\
\hline$\geq 135$ & 0 \\
\hline$<135$ & 2 \\
\hline \multicolumn{2}{|l|}{ Creatinine, $\mu \mathrm{mol} / \mathrm{L}$} \\
\hline$\leq|4|$ & 0 \\
\hline$>|4|$ & 2 \\
\hline \multicolumn{2}{|l|}{ Glucose, $\mathrm{mmol} / \mathrm{L}$} \\
\hline$\leq 10$ & 0 \\
\hline$>10$ & 1 \\
\hline \multicolumn{2}{|l|}{ Wall criteria } \\
\hline $\begin{array}{l}\text { Total white blood cell count }>15.4 \times 10^{9} / \mathrm{L} \\
\text { OR }\end{array}$ & NSTI \\
\hline Sodium $<135 \mathrm{mmol} / \mathrm{L}$ & NSTI \\
\hline
\end{tabular}

Notes: The LRINEC score was described by Wong et $\mathrm{a}^{22}$ and the Wall criteria were described by Wall et al. ${ }^{21} \mathrm{~A}$ LRINEC score of $\geq 6$ was considered predictive for NSTI. The presence of either Wall criteria on admission was considered predictive of NSTI.

Abbreviations: LRINEC, Laboratory Risk Indicator for Necrotizing Fasciitis; NSTI, necrotizing soft tissue infection; OR, odds ratio.

patients received antibiotics, of which $70 \%$ were ordered by the emergency department (vs the admitting service). Median (IQR) time to antibiotic administration was 3.5 hours $(1.9,7.5)$. Fifty-five (92\%) patients had wound cultures, of which $75 \%$ were positive and $33 \%$ were polymicrobial. Polymicrobial synergistic infection was the most common cause (18 patients). Group A streptococcus was the most common cause of monomicrobial NSTI ( 9 patients). Fifty (83\%) patients underwent surgery, with a median (IQR) time from initial presentation to surgery of 15.5 hours $(7.8,74.9)$. Thirteen $(21.6 \%)$ patients died while in hospital. In-hospital mortality among those who had surgical intervention was $14 \%$, compared to $60 \%$ for those who did not receive surgical intervention $(\Delta 46 \%$; $95 \% \mathrm{CI}$ : $14.8 \%-70.2 \%)$.

Seventeen (28.3\%) patients presented with NSTI involving the pelvis, which was the most common body region affected (Table 3). Involvement of the lower extremity occurred in $16(26.7 \%)$ patients, upper extremity in 10 (16.7\%) patients, trunk in 14 (23.3\%) patients, and multiple
Table 2 Summary of demographics, presentation, and outcomes for the 60 cases of NSTI studied

\begin{tabular}{ll}
\hline Variable & Study result \\
\hline Patient demographics & \\
Mean age, years (SD) & $53.7(17.8)$ \\
Sex, male & $60 \%$ \\
Immune compromise & $58 \%$ \\
Vascular disease & $45 \%$ \\
Diabetes mellitus & $42 \%$ \\
Obesity & $25 \%$ \\
Initial presentation & \\
Swelling & $92 \%$ \\
Erythema & $87 \%$ \\
Bruising & $45 \%$ \\
Bullae & $28 \%$ \\
Petechiae & $8 \%$ \\
Location & \\
Upper extremity & $17 \%$ \\
Lower extremity & $55 \%$ \\
Trunk & $23 \%$ \\
Multiple sites & $5 \%$ \\
Outcomes & \\
Median (IQR) length of hospital stay & 17.1 days (7.8, 33.5) \\
In-hospital mortality with surgery & $14 \%$ \\
In-hospital mortality without surgery & $60 \%$ \\
\hline
\end{tabular}

Abbreviations: IQR, interquartile range; NSTI, necrotizing soft tissue infection.

Table 3 Frequency and mortality of NSTIs by body region

\begin{tabular}{llll}
\hline Body region & All patients & Survivors & Nonsurvivors \\
\hline Total & $60(100)$ & $47(78.3)$ & $13(21.6)$ \\
Lower extremity & $16(26.7)$ & $13(81.3)$ & $3(18.8)$ \\
Upper extremity & $10(16.7)$ & $9(90.0)$ & $1(10)$ \\
Pelvis & $17(28.3)$ & $14(82.4)$ & $3(17.6)$ \\
Trunk & $14(23.3)$ & $11(78.6)$ & $3(21.4)$ \\
Multiple regions & $3(5)$ & $0(0)$ & $3(100)^{*}$ \\
\hline
\end{tabular}

Notes: *Significant difference from the expected proportion. Values are displayed as $n(\%)$.

Abbreviation: NSTIs, necrotizing soft tissue infections.

regions in $3(5 \%)$ patients. Mortality was $100 \%$ in those patients with multiple sites affected. For patients presenting with trunk, pelvic, lower extremity, and upper extremity involvement, the mortality rates were $21.4 \%, 17.6 \%, 18.8 \%$, and $10 \%$, respectively (Table 3 ). These were not significantly different from expected proportions (Fisher's exact). Mortality was $100 \%$ in those patients with multiple sites affected $(p<0.05)$.

Laboratory results for serum sodium and WBC were consulted to determine if the Wall criteria ${ }^{21}$ (either WBC count $>15.4 \times 10^{9} / \mathrm{L}$ or serum sodium concentration $<135$ $\mathrm{mmol} / \mathrm{L}$ ) were met for NSTI patients. Forty-four patients met one or both criteria for NSTI, and medical record data were insufficient to assess three patients. The remaining 13 patients did not meet the Wall criteria for NSTI. Therefore, in our study, the criteria laid out by Wall et al had a sensitiv- 
ity of $77 \%$ in those patients for which sufficient data were available. Calculation of the LRINEC score was done with the additional laboratory results for creatinine, glucose, hemoglobin, and CRP. Of the 60 patients, the medical records for only 19 contained enough data to calculate the LRINEC score. CRP levels were most commonly absent. Of these 19 patients, 15 had a LRINEC score of $\geq 6$ (79\%). For patients with incomplete laboratory values, we calculated the theoretical maximum and minimum LRINEC scores by substituting each missing value with the maximum or minimum score for that criterion. By assuming the maximum values for missing data, we found that $51(85 \%)$ of patients potentially had a score of $\geq 6$. Conversely, by assuming the minimum values, we found that only 27 (45\%) of patients scored $\geq 6$.

\section{Discussion}

Results from our study indicate that the most common signs and symptoms of NSTIs were swelling and erythema. Furthermore, prompt, aggressive, and repeated surgical debridement was critical in the treatment of NSTIs. Comorbidities most commonly associated with NSTI were immune compromise, diabetes mellitus, and vascular disease. These findings are consistent with previous studies, $4,11,13,16,20,27$ where the most common presentation of NSTI was one of nonspecific nature. Moreover, previously reported scoring systems $^{21,22}$ for detecting NSTIs were only moderately sensitive in our cohort. The specificities of these systems were not assessed here. However, based on the literature, ${ }^{21,22,24,26,28}$ the strength of these scoring systems may be in their specificity for NSTIs instead of their sensitivity. Consequently, the initiation of appropriate laboratory tests and prompt diagnosis of NSTIs depends heavily on a high index of suspicion on the part of the physician. This is illustrated in our cohort where CRP, the most predictive and influential component of the LRINEC score, ${ }^{22,28}$ was available for only 19 patients. Recently, Borschitz et al proposed an amended version of the LRINEC tool that included clinical findings (pain, fever, tachycardia, evidence of acute renal failure) as well as addition of fibrinogen and erythrocyte levels. ${ }^{17}$ With these changes, they report a sensitivity of $83 \%$ and a specificity of $90 \%$. Future studies will be needed to validate this amended LRINEC tool. But even with improved accuracy, its use will remain contingent on clinicians' inclination for ordering relatively uncommon tests.

The distribution of NSTIs by body region in past studies has favored the lower extremities and the perineum over the trunk and upper extremities. ${ }^{4,16,19,27,29}$ Our study results reflected this distribution. Anaya et al found that out of 166 patients with NSTIs, mortality was significantly higher for those patients with an affected extremity, but not for other regions. ${ }^{30}$ However, the affected body region did not have a significant influence on mortality in our study. Our results are supported by several recent studies that have found body region to not be predictive of mortality. ${ }^{4,16,29,31}$ The possible exception to this in our cohort were the three patients that presented with multiple body regions affected, where mortality was $100 \%$. This disproportionately high mortality rate was statistically significant. However, due to the low number of patients in this group, caution should be exercised in interpreting this result.

Overall mortality in our cohort was $21.6 \%$, which is in line with previously reported mortality rates. ${ }^{10,20,30,32}$ The significant reduction in mortality for patients who underwent surgical debridement indicates the importance of a high index of suspicion and early referral to surgical services. It has been reported that misdiagnosis of NSTIs occurs in $41 \%-96 \%$ of NSTI presentations, ${ }^{20}$ and delayed surgical debridement has repeatedly been shown to significantly increase the mortality risk..$^{3,4,6,9,20,33}$ This may explain the increased mortality rate in our study among patients who did not undergo surgery. However, we cannot assume that patients who did not receive surgical debridement had comparable prognoses upon presentation. Perhaps the decision to forego surgery was influenced more by goals of care and preexisting/complicating comorbidities than missed diagnoses. Thus, any conclusions on mortality rates with and without surgery should be made with this in mind.

This study has several limitations. This was a small study conducted at a single, urban, academic center, and our findings may not be generalizable to other settings. Due to the retrospective nature of this study, we are limited by what was documented in the patient chart. Unfortunately, this restricts the information that can be gathered about the underlying causes of the NSTIs reported here. We can say that at least four of the patients in our cohort developed a NSTI from a previous surgical wound, three from intravenous drug use, two from arthropod bites, one from a pilonidal abscess, one from a furuncle, and one from reactivation of nocardia disease. The remaining charts did not point to a clear cause. Shaikh et al encountered a similar trend when they examined 331 cases of NSTIs at a tertiary care center. They found no history of injury in $85.5 \%$ of cases reviewed. ${ }^{19}$ The remaining cases pointed to trivial trauma (13\%) and intramuscular injections $(1.5 \%)$. This tendency for NSTIs to arise without an obvious cause, or from a trivial injury, further underpins the difficulty in accurate diagnosis. 


\section{Conclusion}

Primary care physicians are often the first to evaluate patients with NSTIs and as such should be aware of the presentation and management of this disease. This study confirmed that diabetes mellitus, immune compromise, vascular disease, and obesity are common comorbidities of NSTIs, and that survival is higher among patients who receive surgical treatment. Patients meeting this clinical picture warrant a high degree of suspicion to avert potentially disastrous consequences.

\section{Acknowledgments}

The authors would like to thank Christopher G Elliott, MD, $\mathrm{PhD}$, for assistance with manuscript preparation. This abstract was presented as a poster presentation at the Society for Academic Emergency Medicine National Annual Meeting in San Diego, May 2015, and published in Academic Emergency Physician on the Wiley Online Library.

\section{Disclosure}

The authors report no conflicts of interest in this work.

\section{References}

1. Anaya DA, Dellinger EP. Necrotizing soft-tissue infection: diagnosis and management. Clin Infect Dis. 2007;44(5):705-710.

2. Puvanendran R, Huey JC, Pasupathy S. Necrotizing fasciitis. Can Fam Physician. 2009;55(10):981-987.

3. Mok MY, Wong SY, Chan TM, Tang WM, Wong WS, Lau CS. Necrotizing fasciitis in rheumatic diseases. Lupus. 2006;15(6):380-383.

4. Kalaivani V, Hiremath BV, Indumathi VA. Necrotising soft tissue infection - risk factors for mortality. J Clin Diagn Res. 2013;7(8): $1662-1665$.

5. Bisno AL, Cockerill FR, Bermudez CT. The initial outpatient-physician encounter in group a streptococcal necrotizing fasciitis. Clin Infect Dis. 2000;31(2):607-608.

6. Wong CH, Chang HC, Pasupathy S, Khin LW, Tan JL, Low CO. Necrotizing fasciitis: clinical presentation, microbiology, and determinants of mortality. J Bone Joint Surg Am. 2003;85-A(8):1454-1460.

7. Kwan, MK, Saw A, Chee EK, et al. Necrotizing fasciitis of the lower limb: an outcome study of surgical treatment. Med J Malaysia. 2006;61(Suppl A):17-20.

8. Ozalay M, Ozkoc G, Akpinar S, Hersekli MA, Tandogan RN. Necrotizing soft-tissue infection of a limb: clinical presentation and factors related to mortality. Foot Ankle Int. 2006;27(8):598-605.

9. Roje Z, Roje Z, Matić D, Librenjak D, Dokuzović S, Varvodić J. Necrotizing fasciitis: literature review of contemporary strategies for diagnosing and management with three case reports: torso, abdominal wall, upper and lower limbs. World J Emerg Surg. 2011;6(1):46.

10. Hakkarainen TW, Kopari NM, Pham TN, Evans HL. Necrotizing soft tissue infections: review and current concepts in treatment, systems of care, and outcomes. Curr Probl Surg. 2014;51(8):344-362.

11. Mitchell A, Williams A, Dzendrowskyj P. Necrotising fasciitis: an 8.5year retrospective case review in a New Zealand intensive care unit. Crit Care Resusc. 2011;13(4):232-237.

12. Davoudian P, Flint NJ. Necrotizing fasciitis. CEACCP. 2012;12:245-250.
13. Sin F, Yuen M, Lam K, Wu CW, Tung WK. A retrospective review of patients with necrotizing fasciitis presenting to an emergency department in Hong Kong. Hong Kong J Emerg Me. 2002;9(1):10-17.

14. Paz Maya S, Dualde Beltrán D, Lemercier P, Leiva-Salinas C. Necrotizing fasciitis: an urgent diagnosis. Skeletal Radiol. 2014;43(5):577-589.

15. Stevens DL, Biosno AL, Chambers HF, et al. Practice guidelines for the diagnosis and management of skin and soft tissue infections: 2014 update by the infectious diseases society of America. Clin Infect Dis. 2014;59(2):147-159.

16. Jabbour G, El-Menyar A, Peralta R, et al. Pattern and predictors of mortality in necrotizing fasciitis patients in a single tertiary hospital. World J Emerg Surg. 2016;11:40.

17. Borschitz T, Schlicht S, Siegel E, Hanke E, von Stebut E. Improvement of a clinical score for necrotizing fasciitis: "pain out of proportion" and high CRP levels aid the diagnosis. PLoS One. 2015;10(7):e0132775

18. Naqvi GA, Malik SA, Jan W. Necrotizing fasciitis of the lower extremity: a case report and current concept of diagnosis and management. Scand J Trauma Resusc Emerg Med. 2009;17:28.

19. Shaikh N, El-Menyar A, Mudali IN, Tabeb A, Al-Thani H. Clinical presentations and outcomes of necrotizing fasciitis in males and females over a 13-year period. Ann Med Surg (Lond). 2015;4(4):355-360.

20. Goh T, Goh LG, Ang CH, Wong CH. Early diagnosis of necrotizing fasciitis. Br J Surg. 2014;101(1):e119-e125.

21. Wall DB, Klein SR, Black S, de Virgilio C. A simple model to help distinguish necrotizing fasciitis from nonnecrotizing soft tissue infection. J Am Coll Surg. 2000;191(3):227-231.

22. Wong $\mathrm{CH}$, Khin LW, Heng KS, Tan KC, Low CO. The LRINEC (Laboratory Risk Indicator for Necrotizing Fasciitis) score: a tool for distinguishing necrotizing fasciitis from other soft tissue infections. Crit Care Med. 2004;32(7):1535-1541.

23. Tsai YH, Hsu RW, Huang KC, Huang TJ. Laboratory indicators for early detection and surgical treatment of vibrio necrotizing fasciitis. Clin Orthop Relat Res. 2010;468(8):2230-2237.

24. Thomas AJ, Meyer TK. Retrospective evaluation of laboratory-based diagnostic tools for cervical necrotizing fasciitis. Laryngoscope. 2012;122(12):2683-2687.

25. Wilson MP, Schneir AB. A case of necrotizing fasciitis with a LRINEC score of zero: clinical suspicion should trump scoring systems. J Emerg Med. 2013;44(5):928-931.

26. Liao C, Lee Y, Su Y, Chuang C, Wong C. Validation of the laboratory risk indicator for necrotizing fasciitis (LRINEC) score for early diagnosis of necrotizing fasciitis. Tzu Chi Med J. 2012;24:73-76.

27. Misiakos EP, Bagias G, Papadopoulos I, et al. Early diagnosis and surgical treatment for necrotizing fasciitis: a multicenter study. Front Surg. 2017;4:5.

28. Narasimhan V, Ooi G, Weidlich S, Carson P. Laboratory Risk Indicator for Necrotizing Fasciitis score for early diagnosis of necrotizing fasciitis in Darwin. ANZ J Surg. 2017.

29. Khamnuan P, Chongruksut W, Jearwattanakanok K, Patumanond J, Yodluangfun S, Tantraworasin A. Necrotizing fasciitis: risk factors of mortality. Risk Manag Healthc Policy. 2015;8:1-7.

30. Anaya DA, McMahon K, Nathens AB, Sullivan SR, Foy H, Bulger E. Predictors of mortality and limb loss in necrotizing soft tissue infections. Arch Surg. 2005;140(2):151-157.

31. Huang KF, Hung MH, Lin YS, et al. Independent predictors of mortality for necrotizing fasciitis: a retrospective analysis in a single institution. J Trauma. 2011;71(2):467-473.

32. Kao LS, Lew DF, Arab SN, et al. Local variations in the epidemiology, microbiology, and outcome of necrotizing soft-tissue infections: a multicenter study. Am J Surg. 2011;202(2):139-145.

33. Boyer A, Vargas F, Coste F. Influence of surgical treatment timing on mortality from necrotizing soft tissue infections requiring intensive care management. Intensive Care Med. 2009;35(5):847-853. 


\section{Publish your work in this journal}

The International Journal of General Medicine is an international, peer-reviewed open-access journal that focuses on general and internal medicine, pathogenesis, epidemiology, diagnosis, monitoring and treatment protocols. The journal is characterized by the rapid reporting of reviews, original research and clinical studies across all disease areas.
The manuscript management system is completely online and includes a very quick and fair peer-review system, which is all easy to use. Visit http://www.dovepress.com/testimonials.php to read real quotes from published authors.

Submit your manuscript here: https://www.dovepress.com/international-journal-of-general-medicine-journal 